\title{
A holey fiber based WDM wavelength converter incorporating an apodized fiber Bragg grating filter
}

\author{
Ju Han Lee, Zulfadzli Yusoff, Walter Belardi, Morten Ibsen, \\ Tanya M. Monro, Benn Thomsen, and David J. Richardson \\ Optoelectronics Research Centre, University of Southampton, Southampton, SO17 1BJ, United Kingdom
}

\begin{abstract}
We demonstrate a tunable WDM wavelength converter based on XPM in just 6m of highly nonlinear holey fiber and incorporating a precise, compression tuned apodized fiber-grating filter. We achieve broadband $10 \mathrm{Gbit} / \mathrm{s}$ wavelength conversion $(\sim 20 \mathrm{~nm})$ with a constant pulsewidth due to short device length and correspondingly reduced pulse walk-off effects.
\end{abstract}

\section{Introduction}

Recent progress in the design and fabrication of holey fiber (HF) has resulted in widespread interest in the use of such technology for a variety of fiber-optic communication and photonics devices and applications [1,2]. The strong wavelength dependence of the effective-index between the core and the cladding, and the possibility of large index contrasts between core and cladding in HF leads to a broader range of optical properties in these fibers than are realizable using conventional fiber fabrication approaches. HF's can have a much higher nonlinearity per unit length than conventional fibers, and consequently devices based on such fibers can be much shorter in length, and/or operate at lower power levels than their conventional counterparts. For example, we have demonstrated a regenerative all-optical switch based on SPM and spectral filtering in just $3.3 \mathrm{~m}$ of HF [1]. We also showed that it was possible to obtain $\sim 42 \mathrm{~dB}$ signal gain in an $\mathrm{L}^{+}$-band Raman amplifier based on just $75 \mathrm{~m}$ length of HF [2]. Recently results on the operation of a nonlinear optical loop mirror switch based on cross-phase modulation (XPM) in a $5.8 \mathrm{~m} \mathrm{HF}$ have been reported [3].

In this paper, we show that HF technology can be applied to provide another important nonlinear function required within wavelength division multiplexing (WDM) systems - namely tunable WDM wavelength conversion. We demonstrate multiple wavelength conversion over $\sim 20 \mathrm{~nm}$ bandwidth at a data rate of $10 \mathrm{Gbit} / \mathrm{s}$ using a combination of XPM in a short length of HF and narrowband spectral filtering. As well as demonstrating a much shorter device in contrast to previously reported wavelength conversion based on the XPM and filtering [4,5], we also use a compression-tuned narrowband apodized fiber Bragg grating (FBG) filter to obtain accurate, high quality spectral definition of the wavelength converted signal. The use of an apodized grating filter allows for a very good extinction of the associated residual continuous wave (CW) and pump beams, as well as for adjacent wavelength converted channels.

\section{Experimental results}

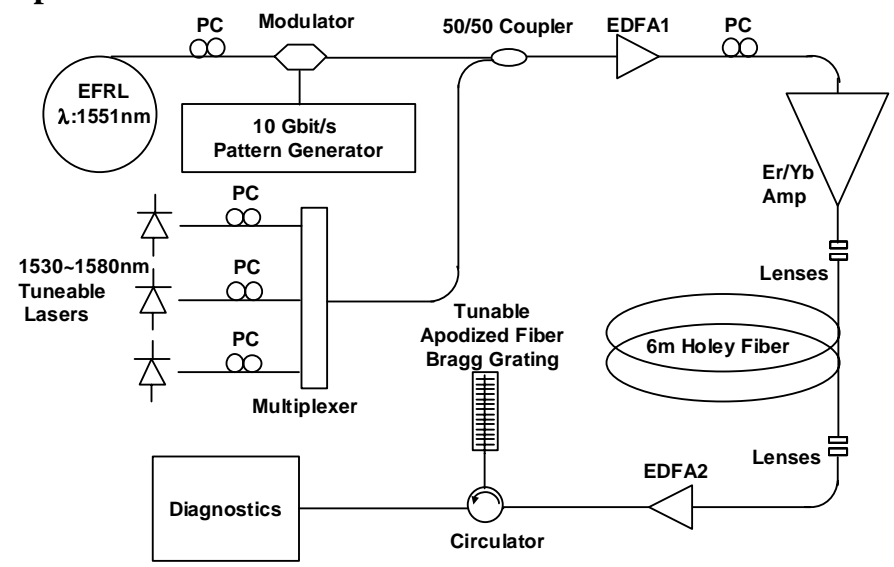

(a)

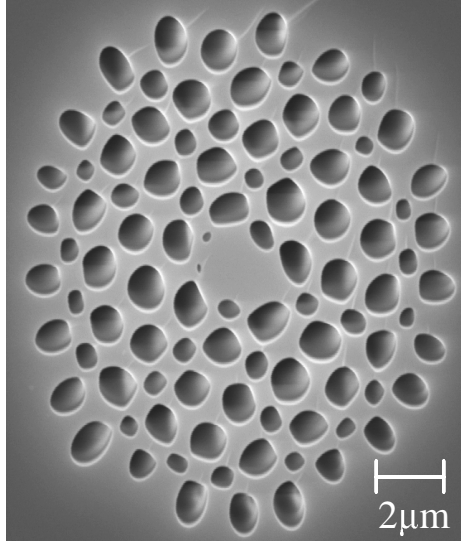

(b)

Fig.1. (a) Experimental setup. (b) Cross-sectional SEM image of the holey fiber used. 
Our experimental set up is shown in Fig. 1(a). 2.5ps pulses at a repetition rate of $10 \mathrm{GHz}$ are first generated using a regeneratively mode locked erbium fiber ring laser (EFRL) operating at a wavelength of $1551 \mathrm{~nm}$. These pulses are then modulated to obtain a $10 \mathrm{Gbit} / \mathrm{s}, 2^{15}$ - pseudorandom control pulse stream. We combined the control pulses and three probe beams together using a combination of 50/50 couplers prior to launching the light into the HF. The probe beams were generated from CW external cavity lasers tunable in the range 1530 1580nm. Polarization controllers were included on both the control and probe launching paths into the HF so that all of the beams could be launched onto a single polarization axis of the polarization maintaining HF. Both the control and probe signals were amplified using an $\mathrm{Er} / \mathrm{Yb}$ amplifier with a maximum saturated output power of $\sim 800 \mathrm{~mW}$, and were then lens coupled into a $6 \mathrm{~m}$ length of HF with a coupling efficiency of $40 \%$. A cross-sectional SEM profile of the highly nonlinear HF used in this experiment is shown in Fig. 1(b). The core diameter is $\sim 2.0 \mu \mathrm{m}$ and the outer diameter is $125 \mu \mathrm{m}$. The measured dispersion at the wavelength of $1550 \mathrm{~nm}$ was $+100 \mathrm{ps} / \mathrm{nm}-\mathrm{km}$ and the measured loss was $50 \mathrm{~dB} / \mathrm{km}$. From a measurement of the SPM induced nonlinear phase shift versus launched optical power, we obtained an estimated value of $\gamma=31 \mathrm{~W}^{-1} \cdot \mathrm{km}^{-1}$ for the nonlinearity coefficient and an estimated effective area of $\mathrm{A}_{\mathrm{eff}}=2.93(+/-0.3) \mu \mathrm{m}^{2}$. This nonlinearity is $\sim 20$ times higher than that of a conventional dispersion shifted fiber.

XPM between the control signal and the CW beams results in chirping of the CW laser beam where these beams overlap temporally within the fiber. This frequency chirping can then be converted to a frequency converted signal by passing this chirped signal through a narrowband filter which serves to eliminate the residual unchirped $\mathrm{CW}$ signal as well as to select one of the two XPM-induced side bands [5]. The measured output spectrum from the HF is shown in Fig. 2(a). It is clearly evident that each probe beam is spectrally broadened due to XPM with the control signal, whilst the control signal itself also shows a small amount of spectral broadening caused by self-phase modulation. In our experiment, we used an apodized FBG for the spectrum-slicing filter. The filter has a $3 \mathrm{~dB}$ bandwidth of $0.55 \mathrm{~nm}$ and a $30 \mathrm{~dB}$ bandwidth of $1.5 \mathrm{~nm}$ as shown in the inset of Fig. 2(b). The FBG was embedded in a layered flexible plate that when bent allowed for $20 \mathrm{~nm}$ wavelength tuning of the filter due to the resulting compressive strain on the FBG [6]. The FBG provides both high precision and high efficiency spectral slicing as well as significant suppression of all residual CW components, pump beams and adjacent wavelength channels as shown in shown in the spectrum of Fig. 2(b).

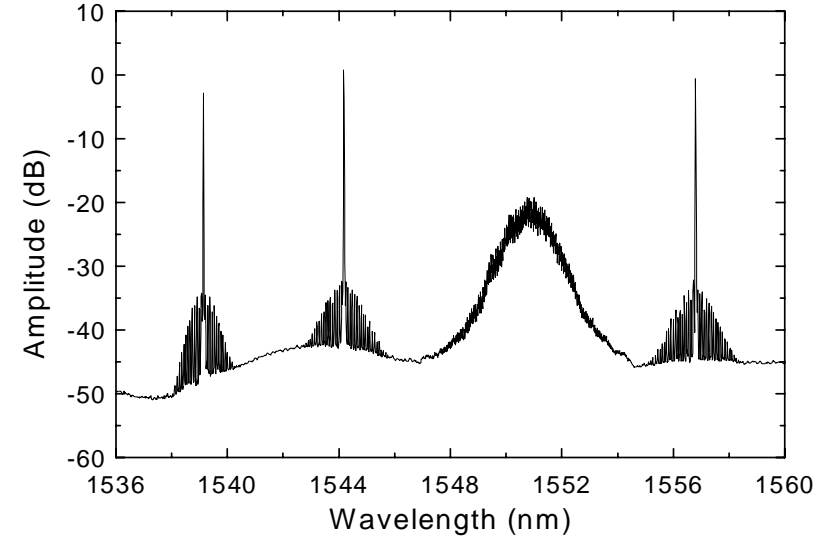

(a)

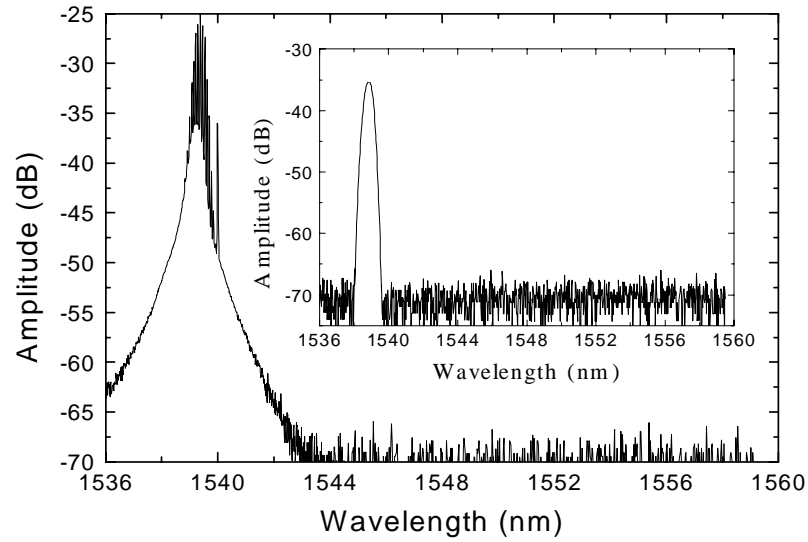

(b)

Fig.2. (a) Measured optical spectrum of three probe signals with control pulses after the HF. (b) Measured optical output spectrum after HF and the grating filter; Inset: Spectral response of the apodized fiber Bragg grating filter used (3dB bandwidth of $0.55 \mathrm{~nm}, 30 \mathrm{~dB}$ bandwidth of $1.5 \mathrm{~nm}$ )

Initially we characterized the performance of our wavelength converter in the temporal domain using an SHG autocorrelator $(<100 \mathrm{fs}$ resolution). The measured temporal width of the wavelength-converted pulses as a function of probe beam wavelength is plotted in Fig. 3(a). The pulsewidths of the converted pulses were observed to be almost constant at $\sim 11 \mathrm{ps}$ over the full, $\sim 20 \mathrm{~nm}$ wavelength tuning wavelength range of our FBG, for any walk-off effect between the control and probe light beams had negligible impact due to the short length of HF used. The timebandwidth product of the converted pulses was $\sim 0.5$ which, although somewhat larger than that of the original control soliton pulses (0.32, transform-limited), still indicates high-quality pulses. However, a degree of amplitude noise was observed on the converted signals and which can be attributed to coherence degradation due to the anomalous dispersion of this particular HF [7]. (This noise could be eliminated by the use of an HF with normal dispersion at the operating wavelength rather than anomalous dispersion. The production of such fibers could be achieved quite easily by relatively minor changes to the structure of the existing HF.) It is well known that the use of a saturable absorber can also reduce intensity noise to a significant extent [8] and so we incorporated a 
semiconductor optical amplifier (SOA) in our experimental setup to help eliminate this amplitude noise, and to allow us to make some measurements of the HF based wavelength converter. We performed BER measurements on one channel at a wavelength of $1540 \mathrm{~nm}$. Fig. 3(b) shows both the measured BER and eye diagrams for the soliton control and wavelength converted $10 \mathrm{Gbit} / \mathrm{s}$ pulses. We observed an approximately $4 \mathrm{~dB}$ power penalty and an error floor at the $10^{-9}$ BER level relative to that of the soliton control pulses. We point out again though that the use of normal dispersion fiber would have eliminated these coherence related penalties.

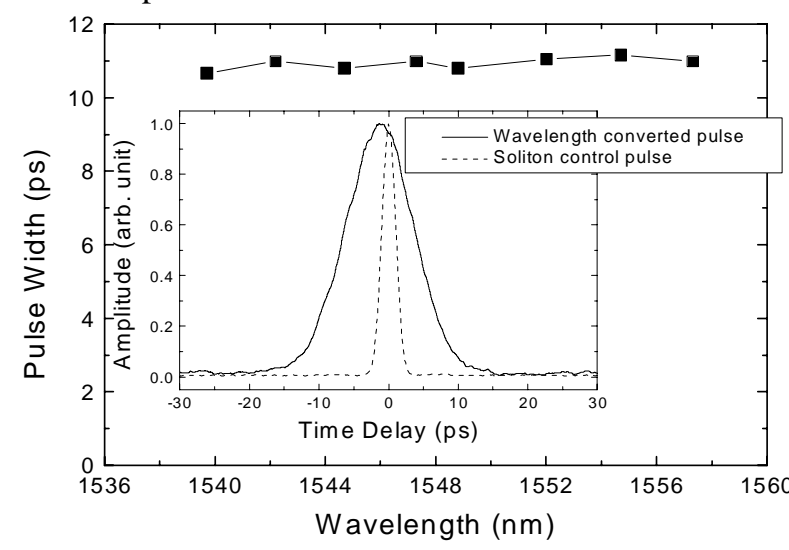

(a)

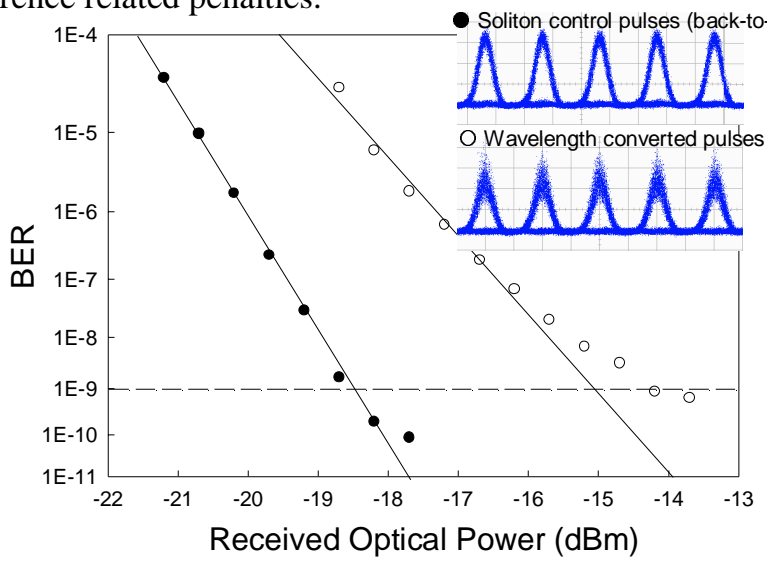

(b)

Fig.2. (a) Measured FWHM of wavelength converted pulses as a function of probe beam wavelength at a single channel case; Inset: SHG autocorrelation traces of wavelength converted pulses at $1540 \mathrm{~nm}$. (b) Measured BER versus received optical power for wavelength conversion of $10 \mathrm{Gbit} / \mathrm{s}$ data pulses at a wavelength of $1540 \mathrm{~nm}$.

\section{Conclusions}

We have experimentally demonstrated a HF based WDM wavelength converter using XPM and spectral filtering. A combination of just $6 \mathrm{~m}$ of highly nonlinear $\mathrm{HF}$ and an apodized fiber Bragg grating filter with a high extinction ratio spectral splicing capability enabled us to achieve simultaneous wavelength conversion of multiple channels over a $\sim 20 \mathrm{~nm}$ bandwidth at a data rate of 10Gbit/s, with almost perfect suppression of the residual CW light as well as negligible pulse width variation due to pulse walk-off effects. The use of a HF with normal dispersion to reduce coherence degradation should allow for the development of practical, all-fiber, and high performance WDM wavelength converters. Ultimately, HF technology should prove to be a powerful way for the realization of a wide range of practical nonlinear optical devices for fiber-optic communication systems.

The authors would like to thank Mr. M. R. Mokhtar for the use of his FBG and associated help.

\section{References}

[1] P. Petropoulos, T.M. Monro, W. Belardi, K. Frusawa, J.H. Lee, and D.J. Richardson, "2 R -regenerative alloptical switch based on a highly nonlinear holey fiber," Optics Lett., 26, 1233-1235 (2001).

[2] Z. Yusoff, J.H. Lee, W. Belardi, T.M. Monro, P.C. Teh, and D.J. Richardson, "Raman effects in a highly nonlinear holey fiber: Amplification and Modulation," Optics Lett., 27, pp.424-426 (2002).

[3] J. E. Sharping, M. Fiorentino, P. Kumar, and R. S. Windeler, "All-optical switching based on cross-phase modulation in microstructure fiber," IEEE Photon. Technol. Lett., 14, pp.77-79 (2002).

[4] J. Yu, and P. Jeppesen, "80-Gb/s wavelength conversion based on cross-phase modulation in high-nonlinearity dispersion-shifted fiber and optical filtering," IEEE Photon. Technol. Lett., 13, pp.833-835 (2001).

[5] P. Ohlen, B. -E. Olsson, and D. Blumenthal, "Wavelength dependence and power requirements of a wavelength converter based on XPM in a dispersion-shifted optical fiber," IEEE Photon. Technol. Lett., 12, pp.522-524 (2000).

[6] S. Y. Set, B. Dabarsyah, C. S. Goh, K. Katoh, Y. Takushima, K. Kikuchi, Y. Okabe, and N. Takeda, "A widely tunable fiber Bragg grating with a wavelength tenability over 40nm," OFC'2001 Anaheim 2001 MC4.

[7] N. Nakazawa, H. Kubota, and K. Tamura, "Random evolution and coherence degradation of a high-order optical soliton train in the presence of noise," Optics Lett., 24, pp.318-320 (1999).

[8] S.-J. Kim, J.-H. Han, J.-S. Lee, and C.-S. Park, "Intensity noise suppression in spectrum-sliced incoherent light communication systems using a gain-saturated semiconductor optical amplifier," IEEE Photon. Technol. Lett., 11, pp.1042-1044 (1999). 